\title{
PdPt@Au Core@Shell Nanoparticles: Alloyed-core Manipulation of CO Electrocatalytic Oxidation Properties
}

\author{
Xinliang Li, Xinlin Hong* \\ College of Chemistry and Molecular Sciences, Wuhan University, Wuhan 430072, P. R. China. \\ * To whom correspondence should be addressed: hongxl@whu.edu.cn
}

\begin{abstract}
A series of PdPt@Au core@shell nanopaticles (NPs) of similar size are synthesized with composition controlled cores and thickness controlled shells. Their $\mathrm{CO}$ electrocatalytic oxidation properties change with the composition of the cores, showing a volcano curve. A correlation between the properties of $\mathrm{CO}$ electrocatalytic oxidation and the location of adsorbed CO ATR-IR peak is discovered. Attenuated Total Reflection-Infrared Spectroscopy (ATR-IR) shows that samples which have the strongest monodentate $\mathrm{CO}$ is most active in $\mathrm{CO}$ electrocatalytic oxidation.
\end{abstract}

\section{Keywords}

core@shell,PdPt@Au, CO electrocatalytic oxidation, ATR-IR

\section{Introduction}

Polymetallic nanoparticles (NPs) are not only a simple combination of two distinct metals but also expected to display new properties and capabilities due to a synergy between different metals. [1, 2, 3] More importantly, core@shell nanoparticles usually show composition-dependent surface structure and atomic segregation behavior. $[4,5,6]$ Due to their novel catalytic [7, 8], electronic [9, 10, 11, 12, 13] and magnetic properties [14, 15,16], core@shell nanopaticles attracted more attention in these years. However, preparation of core@shell nanoparticles with ideal monodispersity still is a challenge, and ideal crystal face that is much different from the industrial catalyst is usually used by researchers.[17] In this work, we made model catalysts that are closer to real catalysts to explore the effect of the core and shell. A series of PdPt@Au core@shell nanoparticles of similar size were synthesized with composition controlled cores and thickness controlled shells. They show a volcano curve in electrocatalytic oxidation of CO. And from the Attenuated Total Reflection-Infrared Spectroscopy (ATR-IR) spectra of $\mathrm{CO}$ adsorption, we can see the strongest monodentate $\mathrm{CO}$ is most beneficial to the $\mathrm{CO}$ electrocatalytic oxidation. Furthermore, a correlation between the activity of $\mathrm{CO}$ electrocatalytic oxidation and the location of adsorbed CO ATR-IR peak is discovered.

\section{Experimental}

\subsection{Synthesis of the samples}

The PdPt@Au NPs were synthesized through a seeded-growth method consisting of two steps. (See the Supporting materials for details) Firstly, the core seeds that stabilized by PVP were synthesized in the solvent of ethylene glycol. Then, they were transferred into $\mathrm{H}_{2} \mathrm{O}$ to grow the Au shell. Before further study, the samples were washed with ethanol and ultrapure water by repeating the centrifugation and re-dispersion procedure several times to remove the excess PVP, chloride ions and other impurities. Then, these washed core@shell NPs were supported on carbon black Vulcan XC-72 (20 wt\%). (See the Supporting materials for details) The deposited NPs were washed extensively with ethanol and ultrapure water to remove any residual chloride ions and PVP before 
electrochemical measurements.

\subsection{Characterization}

Powder X-ray diffraction (XRD) data was collected on a Shimadzu XRD-6000 X-ray diffractometer using a Cu Ka radiation source operating at $40 \mathrm{kV}$ and $30 \mathrm{~mA}$. Transmission electron microscopy (TEM) observations were collected on a JEOL JEM-2100 TEM. High-angle annular dark-field scanning transmission electron microscopy (HAADF-STEM) observations were collected on a JEOL JEM-2100F TEM. Attenuated Total Reflection-Infrared Spectroscopy (ATR-IR) spectra were acquired using a Bruker Vertex 70 with a liquid-nitrogen-cooled MCT detector.

\subsection{CO electrocatalytic oxidation tests}

The catalytic activity of the carbon supported core@shell NPs in the electroxidation of CO was examined by voltammetric measurements. Electrochemical studies were carried out in a standard three-electrode cell connected to a $\mathrm{CHI}-440$ electrochemical workstation, with a Pt foil counter electrode and a standard calomel electrode (SCE) at room temperature in $0.1 \mathrm{M} \mathrm{HClO}_{4}$ solution. To prepare the working electrode, $5 \mathrm{mg}$ carbon-supported core@shell NPs was dispersed ultrasonically in $1 \mathrm{~mL} 5$ wt\% Nafion solution to form an ink, and $10 \mu \mathrm{L}$ of the ink was pipetted onto a glassy carbon $(\mathrm{GC})$ substrate $(\Phi=5 \mathrm{~mm})$, which had been buff-polished with an alumina suspension $(\Phi=0.05 \mu \mathrm{m})$ prior to use. The catalyst-coated electrode was dried under an infrared lamp.

Before executing the $\mathrm{CO}$ oxidation by positive-going linear sweep voltammetry, the electrolyte was firstly deaerated by bubbling $\mathrm{Ar}$ for $30 \mathrm{~min}$. Then three cycles of voltammetry were run, with potential limits of -0.28 to $0.90 \mathrm{~V}$, to ensure the cleanliness of the sample. In order to adsorb $\mathrm{CO}$ at a saturated coverage on the electrode, $\mathrm{CO}$ was bubbled through the electrolyte for $30 \mathrm{~min}$ while the electrode was under potentiostatic control at $0.20 \mathrm{~V}$. After the $\mathrm{CO}$ adsorption process, dissolved $\mathrm{CO}$ was removed from the electrolyte by bubbling $\mathrm{Ar}$ for $1 \mathrm{~h}$. This process simultaneously ensures a full coverage of $\mathrm{CO}$ on the electrode and complete removal of $\mathrm{CO}$ from the electrolyte. [18] Then, the $\mathrm{CO}$ was electro-oxidized by scanning the potential at $20 \mathrm{mV} / \mathrm{s}$ from $-0.28 \mathrm{~V}$ to $0.90 \mathrm{~V}$, followed by several cycles of voltammetry.

\section{Results and discussion}

\subsection{TEM and HADDF-STEM characterization}

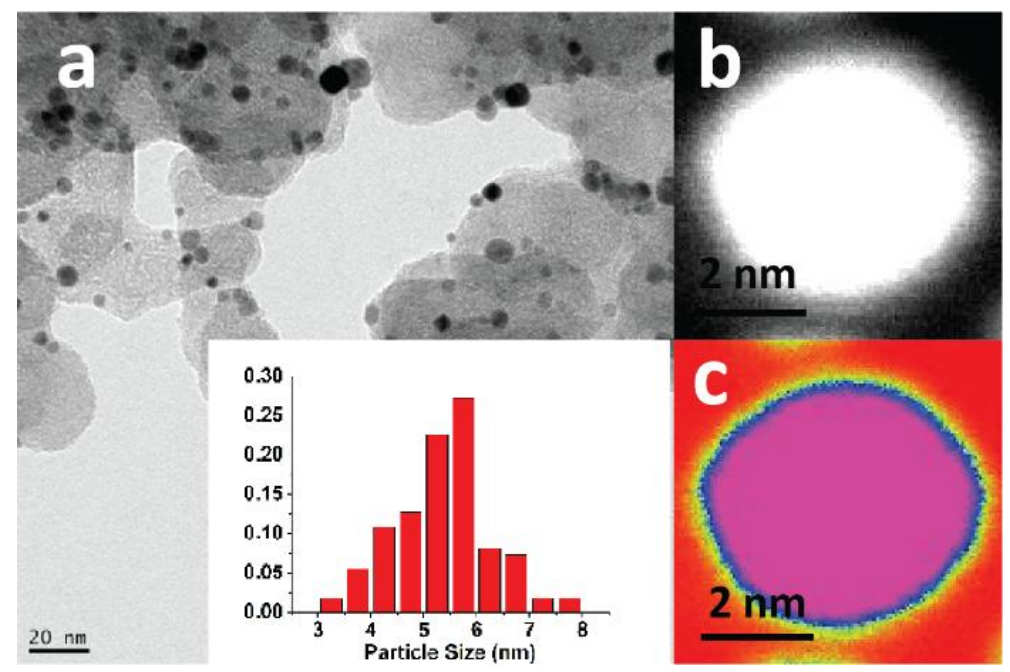

Fig. 1 (a) TEM image of carbon-supported PdPt@Au(Pd:Pt=1:1) NPs. The average size of the sample is 5.36nm. (b) HAADF-STEM image of the sample. (c) Pseudo-colored HAADF-STEM images of the sample. The purple section is the alloy core and the blue section is the Au shell

The TEM image in Fig. 1a indicates that the PdPt@Au(Pd:Pt=1:1) NPs are dispersed uniformly on the carbon support. Most of the NPs are in spherical shape. The size distribution analysis in Fig. 1a shows that the particles 
have an average size of $5.36 \mathrm{~nm}$. A typical HAADF-STEM in Fig. $1 \mathrm{~b}$ shows the alloy core appears to be bright relative to the Au shell, but the Au shell is too thin to get clear contrast. There is limited information due to the monochrome image. So we get the pseudo-colored HAADF-STEM image showing in Fig. 1c according to the monochrome one. [19] From the pseudo-colored HAADF-STEM image, we can easily distinguish the alloy core in purple from the Au shell in blue. In addition, we can find that the core is packed by the Au shell perfectly and the Au shell is only about several atomic layers. The rest four samples are almost the same to $\mathrm{PdPt}(\mathrm{Pd}: \mathrm{Pt}=1: 1) \mathrm{NPs}$ in pseudo-colored HAADF-STEM image. The average sizes of the five samples are arranging from $4.02 \mathrm{~nm}$ to 5.97nm. TEM, HAADF-STEM and pseudo-colored HAADF-STEM images of Pd@Au, PdPt@Au(Pd:Pt=3:1), PdPt@Au(Pd:Pt=1:3) and Pt@Au NPs are in the Supporting materials(Fig. S1-S4).

\subsection{XRD characterization}

The XRD patterns of the alloy seeds without the Au shell are showed in Fig. 2. The data (Fig. 2a) indicate that all of the compositions resulted in a single phase: a solid solution of $\mathrm{Pd}$ and $\mathrm{Pt}$ atoms. And the (111) peaks of Pt, $\mathrm{PdPt}(\mathrm{Pd}: \mathrm{Pt}=1: 3), \mathrm{PdPt}(\mathrm{Pd}: \mathrm{Pt}=1: 1), \mathrm{PdPt}(\mathrm{Pd}: \mathrm{Pt}=3: 1)$ and $\mathrm{Pd} \mathrm{NPs}$ locate separately at $39.22^{\circ}, 39.37^{\circ}, 39.47^{\circ}, 39$. $59^{\circ}, 39.70^{\circ}$. They arrange from $39.22^{\circ}$ to $39.70^{\circ}$, showing a regular pattern in Fig. 2b. Lattice expansion is also found in these XRD patterns for the peak skewing to low angle contrasting to standard XRD cards, as a result of small size. [20]
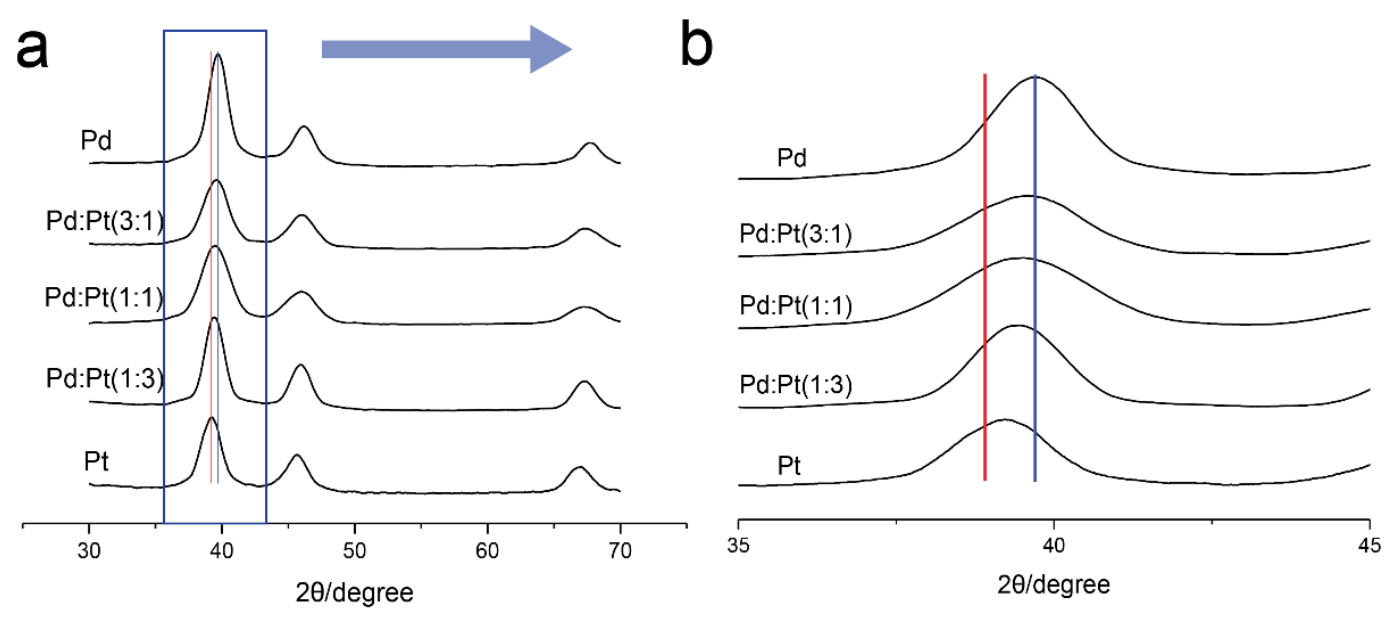

Fig. 2 (a) XRD patterns of the alloy seeds without the Au shell, containing Pd NPs, PdPt(Pd:Pt=3:1) NPs, PdPt(Pd:Pt=1:1) NPs, $\operatorname{PdPt}(\mathrm{Pd}: \mathrm{Pt}=1: 3)$ NPs and Pt NPs. (b) Parts of the XRD patterns from 25 degree to 45 degree.

\subsection{CO electrocatalytic oxidation and AIR-IR tests of $\mathrm{CO}$ adsorption}

The catalytic activity of the carbon supported core@shell NPs and alloy NPs in the electrocatalytic oxidation of CO was examined by voltammetric measurements. The influence of the core composition on the positive-going linear sweep voltammetry turn-on potentials is showed in Fig. S7. (CO-stripping voltammetry curves are showed in Fig. S5, see the Supporting materials for details.) The PdPt@Au/C sample with a core of Pd:Pt=1:1 has the lowest CO stripping turn-on potential. That means the CO electrocatalytic activity of PdPt@Au (Pd:Pt=1:1) sample is superior to that of PdPt@Au samples with other PdPt-ratio cores. And we also can conclude that Pt@Au/C sample is more active than Pd@Au/C sample and PdPt@Au/C samples with Pd-rich cores, but less active than PdPt@Au/C samples with Pt-rich cores. However, the turn-on potential of alloy samples decreases with the increasing of the Pt:Pd ratio, due to the higher activity of Pt.[21]

The spectrum of ATR-IR spectra and data of CO adsorption on core@shell samples and alloy core samples are showed in Fig.S7 (see the Supporting materials for details). For CO on metal surfaces, the CO stretching frequency (2143 $\mathrm{cm}^{-1}$ in the gas-phase) is shifted to $1950-2140 \mathrm{~cm}^{-1}$ when $\mathrm{CO}$ is adsorbed on one metal atom (monodentate CO), to $1800-2000 \mathrm{~cm}^{-1}$ for CO bridging two metal sites (bridging CO). [22] The bridging $\mathrm{CO}$ is a strong adsorption state by contrasted with monodentate $\mathrm{CO}$ which is a weak adsorption state. [23] The adsorption 
intensity grows with the decreasing of the wavenumber. [24] Fig. 3 combines the CO adsorption peak with the results of $\mathrm{CO}$ oxidation by positive-going linear sweep voltammetry, we can see that samples with the bridging $\mathrm{CO}$ (Pd@Au, PdPt@Au(Pd:Pt=3:1) NPs) show relatively low activity in CO oxidation. And the stronger the adsorption of bridging $\mathrm{CO}$, the lower activity in $\mathrm{CO}$ electrocatalytic oxidation reaction. However, samples with the monodentate CO (PdPt@Au(Pd:Pt=1:1),PdPt@Au(Pd:Pt=1:3), Pt@Au NPs) shows relatively high activity. And the stronger adsorption of monodentate $\mathrm{CO}$, the higher activity in $\mathrm{CO}$ electrocatalytic oxidation reaction. In conclusion, the strongest monodentate $\mathrm{CO}$ is most active in $\mathrm{CO}$ electrocatalytic oxidation reaction.

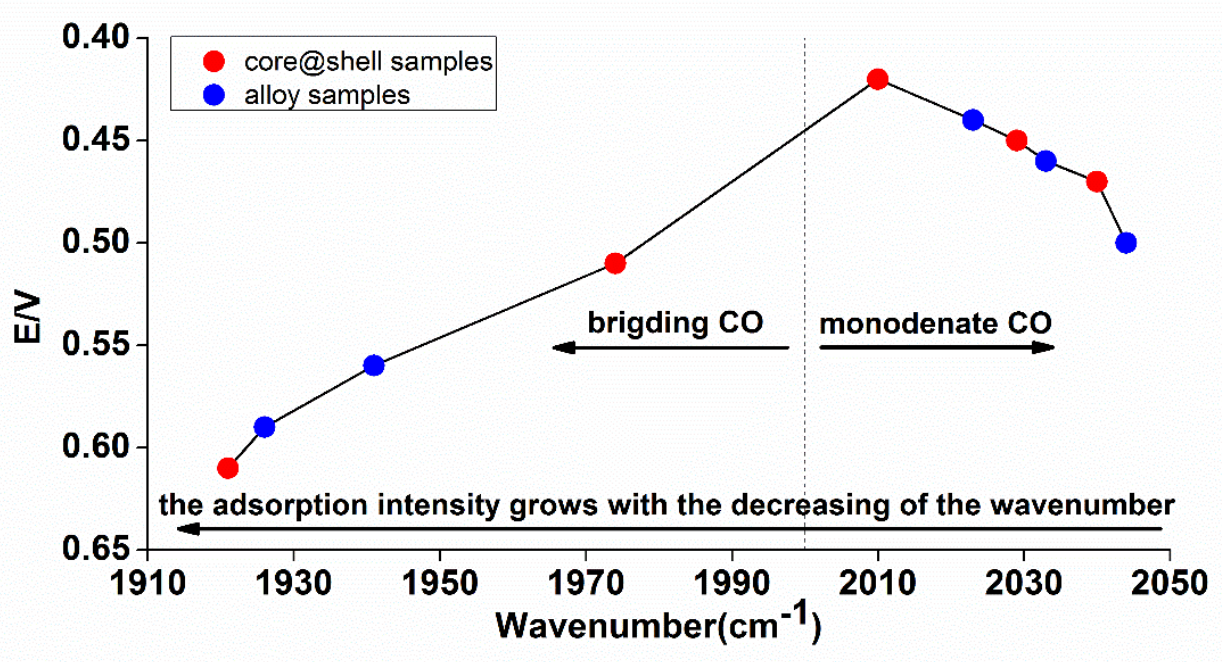

Fig. 3 The correlation between the properties of CO electrocatalytic oxidation and the location of adsorbed CO ATR-IR peak. The $X$ axis shows the positive-going linear sweep voltammetry turn-on potentials and the $Y$ axis shows the wavenumber of adsorbed CO ATR-IR peak

Fig. 4 illustrates the possible electrocatalytic oxidation process of CO on PdPt@Au NPs in acidic electrolyte. Catalysis on metal NPs involves the match between the d-bands center of metal and the energy of adsorbed molecules, such interactions play a critical role in any useful theory of electrochemical bond-breaking reactions. [25] The d-band center of PdPt@Au NPs showing the strongest monodentate CO adsorption may be at an appropriate level to the critical step of CO electrooxidation. [26]

$$
\mathrm{X}-\mathrm{CO}+\mathrm{X}-\mathrm{OH} \rightarrow \mathrm{X}+\mathrm{CO}_{2}+\mathrm{e}^{-}+\mathrm{H}^{+}
$$

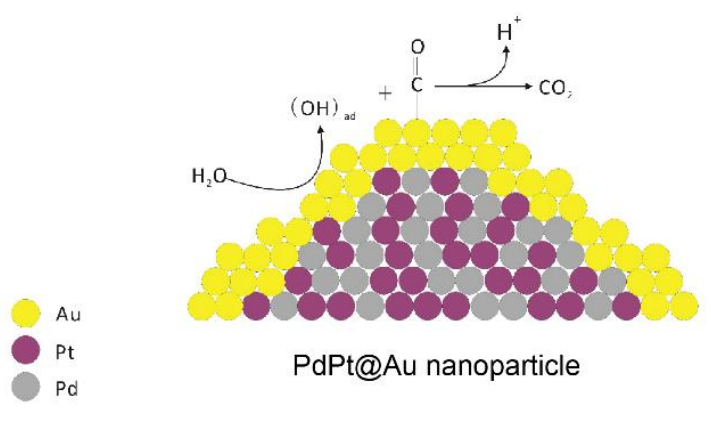

Fig. 4 A possible process of the electrocatalytic oxidation of CO on PdPt@Au NPs catalyst in acidic electrolyte.

\section{Conclutions}

In summary, a series of PdPt@Au core@shell NPs with composition controlled cores and thickness controlled shells 
are synthesized. The study of CO electrocatalytic oxidation and adsorption shows that the PdPt@Au(Pd:Pt=1:1) sample has best $\mathrm{CO}$ electrocatalytic oxidation activity. And the study also indicates that sample which have the strongest monodentate $\mathrm{CO}$ is most active in $\mathrm{CO}$ electrocatalytic oxidation.

\section{Acknowledgements}

This work was financially supported by National Science Foundation of China (NSFC-21373153) and Science Foundation of Jiangsu Province (BK2012638). We would like to thank Prof. Renchao Che of Fudan University for his help in HAADF-STEM measurements, Prof. Shengli Chen (Wuhan University) for his help in CO stripping measurement and Prof. Shik Chi Tsang (University of Oxford) for his constructive advice.

\section{References}

[1] Toshima, N. Yonezawa, T. New J. Chem. 22 (1998) 1179-1201.

URL: http://pubs.rsc.org/en/Content/ArticleLanding/1998/NJ/a805753b

[2] J. A. Rodriguez, D. W. Goodman, Science. 257 (1992) 897-903.

URL: http://science.sciencemag.org/content/257/5072/897.abstract

[3] T. Krenke, E. Duman, M. Acet, E. F. Wassermann, X. Moya, L. Mansa, A. Planes, Nature Mater. 4 (2005) $450-454$. URL: http://www.nature.com/nmat/journal/v4/n6/full/nmat1395.html

[4] Zhang N, Liu S, Xu YJ, Nanoscale. 4 (2012) 2227-2238.

URL: http://pubs.rsc.org/en/Content/ArticleLanding/2012/NR/C2NR00009A

[5] Somorjai, G. A. Park, J. Y., Angew. Chem. Int. Ed. 47 (2008) 9212-9228.

URL: http://onlinelibrary.wiley.com/doi/10.1002/anie.200890245/abstract

[6] Zaera, F. Catal., Lett. 142 (2012) 501-516.

URL:https://www.researchgate.net/publication/257544917_New_Challenges_in_Heterogeneous_Catalysis_for_the_2 1st_Century

[7] M. C. Daniel, D. Astruc, Chem. Rev., 104 (2004) 293-346.

URL: http://www.ncbi.nlm.nih.gov/pubmed/14719978

[8] M. B. Gawande, V. D. B. Bonifacio, R. S. Varma, I. D. Nogueira, N. Bundaleski, C. A. A. Ghumman, O. M. N. D. Teodoro, P. S. Branco, Green Chem. 15 (2013) 1226-1231.

URL: http://www.oalib.com/paper/3090160\#.VtOsJ-bbvCA

[9] A. K. Geim, K. S. Novoselov, Nat. Mater. 6 (2007) 183-191.

URL: http://www.nature.com/nmat/journal/v6/n3/abs/nmat1849.html

[10] V. Georgakilas, A. B. Bourlinos, R. Zboril, T. A. Steriotis, P. Dallas, A. K. Stubos, C. Trapalis, Chem. Commun. 46 (2010) 1766-1768.

URL: http://pubs.rsc.org/en/Content/ArticleLanding/2010/CC/B922081J

[11] V. Georgakilas, M. Otyepka, A. B. Bourlinos, V. Chandra, N. Kim, K. C. Kemp, P. Hobza, R. Zboril, K. S. Kim, Chem. Rev. 112 (2012) 6156-6214.

URL: http://www.col.org.cn/abstract.aspx?id=COL201311083001-03

[12] M. Pykal, K. Safarova, K. M. Siskova, P. Jurecka, A. B. Bourlinos, R. Zboril, M. Otyepka, J. Phys. Chem. C, 117 (2013) 11800-11803.

URL:https://www.researchgate.net/publication/263941872_Lipid_Enhanced_Exfoliation_for_Production_of_Graphen e_Nanosheets

[13] R. Zboril, F. Karlicky, A. B. Bourlinos, T. A. Steriotis, A. K. Stubos, V. Georgakilas, K. Safarova, D. Jancik, C. Trapalis, M. Otyepka, Small. 6 (2010) 2885-2891.

URL: http://onlinelibrary.wiley.com/doi/10.1002/smll.201001401/abstract

[14] M. B. Gawande, P. S. Branco, R. S. Varma, Chem. Soc. Rev. 42 (2013) 3371-3393. 
URL: http://pubs.rsc.org/en/Content/ArticleLanding/2013/CS/C3CS35480F\#!

[15] M. B. Gawande, A. K. Rathi, I. D. Nogueira, R. S. Varma, P. S. Branco, Green Chem. 15 (2013) 1895-1899.

URL: http://pubs.rsc.org/en/Content/ArticleLanding/2013/GC/C3GC36844K

[16] A. H. Lu, E. L. Salabas, F. Schuth, Angew. Chem. Int. Ed. 46 (2007) 1222-1244.

URL: http://onlinelibrary.wiley.com/doi/10.1002/anie.200602866/abstract

[17] D. Wang, Y. Li, Adv. Mater. (2011) 1044-1060.

URL: http://onlinelibrary.wiley.com/doi/10.1002/adma.201003695/full

[18] Mickelson, L. Heaton, T. Friesen, C. J., Phys. Chem. C. 112 (2008) 1060-1063.

URL: www.researchgate.net/publication/231644098

[19] Diehm, Agoston P, Albe K. CHEMPHYSCHEM. 13 (2012) 2443-2454.

URL: http://onlinelibrary.wiley.com/doi/10.1002/cphc.201200257/full

[20] Ilke Arslan, John C. Walmsley, Erling Rytter, Evard Bergene, Paul A. Midgley, J. AM. CHEM. SOC. 130 (2008) 5716-5719.

URL:https://www.researchgate.net/publication/5460042_Toward_Three-Dimensional_Nanoengineering_of_Heteroge neous_Catalysts

[21] In-Su Park, De-Jun Chen, Dianne O. Atienza, YuYe J. Tong., Catalysis Today. 202 (2013) 175-182.

URL: http://www.sciencedirect.com/science/journal/09205861/202

[22] Martin Sterrer, Maxim Yulikov, Thomas Risse, Gianfranco Pacchioni, Angew. Chem. Int. Ed. 45 (2006) 2633-2635.

URL: http://onlinelibrary.wiley.com/doi/10.1002/anie.200504473/abstract

[23] S. Abbet, E. Riedo, H. Brune, U. Heiz, A. M. Ferrari, L. Giordano, G. Pacchioni, J. Am. Chem. Soc. 123 (2001) 6172-6178.

URL: http://infoscience.epfl.ch/record/135797?ln=en

[24] M. Frank, M. Blumer, R. Kuehnemuth, H.-J. Freund, J. Phys. Chem. B. 105 (2001) 8569-8576.

URL:https://www.researchgate.net/publication/260210121_Metal_Atoms_and_Particles_on_Oxide_Supports_Probin g_Structure_and_Charge_by_Infrared_Spectroscopy

[25] Elizabeth Santos, Wolfgang Schmickler, ChemPhysChem. 7 (2006) 2282-2285.

URL: http://onlinelibrary.wiley.com/doi/10.1002/cphc.200600441/full

[26] Takahiro Yajima, Hiroyuki Uchida, Masahiro Watanabe, J. Phys. Chem. B. 108 (2004) 2654-2659.

URL:https://www.researchgate.net/publication/244403359_In-situ_ATR-FTIR_spectroscopic_study_of_electro-oxidati on_of_methanol_and_adsorbed_co_at_Pt-Ru_alloy_J_Phys_Chem_B 


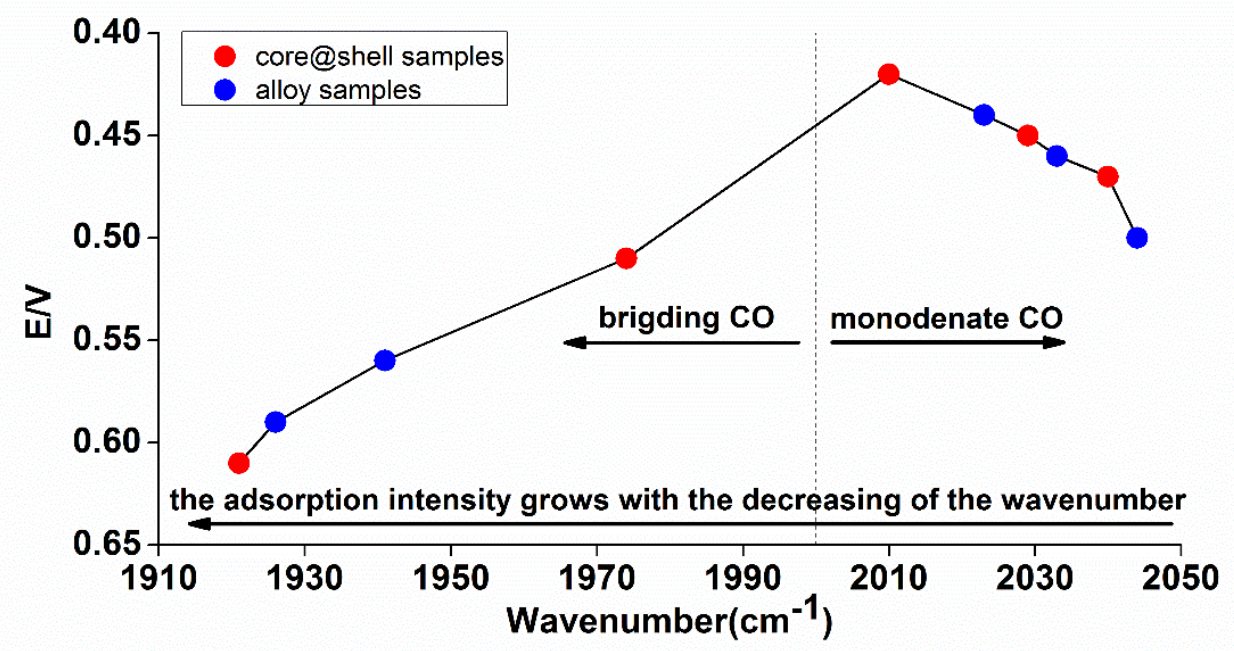

